\title{
A qualitative study on the design and development of an mHealth app to facilitate communication with the Deaf community: perspective of community pharmacists
}

This article was published in the following Dove Medical Press journal:

Patient Preference and Adherence

\author{
Elizabeth Yie-Chuen Chong' \\ Uma Devi Palanisamy² \\ Sabrina Anne Jacob ${ }^{1,3}$ \\ 'School of Pharmacy, Monash \\ University Malaysia, Jalan Lagoon \\ Selatan, Sunway, Selangor, Malaysia; \\ ${ }^{2}$ Jeffrey Cheah School of Medicine and \\ Health Sciences, Monash University \\ Malaysia, Jalan Lagoon Selatan, Sunway, \\ Selangor, Malaysia; ${ }^{3}$ Strathclyde \\ Institute of Pharmacy and Biomedical \\ Sciences, University of Strathclyde, \\ Glasgow, UK
}

Purpose: This study prepares the groundwork on the potential design and development of a mobile health (mHealth) app that will be able to bridge the communication gap between pharmacists and patients who are Deaf and Hard of Hearing (DHoH).

Patients and methods: A focus group discussion was conducted with 12 community pharmacists. Participants were recruited using snowball sampling. Audio-recordings were transcribed verbatim, and analyzed using a thematic approach.

Results: Three themes were apparent: 1) suggestions for app design and content, 2) perceived benefits of the app, and 3) potential challenges related to the app. Participants believed the app would be able to facilitate and improve communication, and hence relationship, between pharmacists and the $\mathrm{DHoH}$. Potential challenges of the app were highlighted, such as the need for manpower to manage the app, and its cost to this group of economically disadvantaged people. There were also concerns about privacy and security.

Conclusions: This study allowed community pharmacists, one of the end-users of the app, to provide feedback on the contents and design of the app, which would allow them to provide pharmaceutical care services to patients who are $\mathrm{DHoH}$, and better serve them. Potential benefits and challenges of the app were also identified. Undoubtedly, through the mHealth app, community pharmacists will be better equipped to serve and communicate with the $\mathrm{DHoH}$, and this will hopefully translate to improved health outcomes in these patients.

Keywords: Deaf, community pharmacist, mHealth, pharmaceutical care, communication, qualitative

\section{Introduction}

According to the 2012 World Health Organization report, it is estimated that 360 million people worldwide live with disabling hearing loss. ${ }^{1}$ For clarity of terminology, the word Deaf is spelt with an uppercase "D" which denotes a minority group who share a cultural identity and a common language - sign language. A number of studies have noted that patients who are Deaf and Hard of Hearing ( $\mathrm{DHoH})$ struggle to access health care due to severe communication barriers. ${ }^{2-6}$ Most health care professionals are linguistically and culturally ill-prepared to provide competent care to Deaf patients, and it has been highlighted that there is lack of knowledge among health care professionals on Deaf culture and their rights. ${ }^{2}$ A focus group discussion (FGD) conducted among 91 Deaf individuals to better understand their health experiences when using American sign language (ASL) found that speech reading is not a viable method of
Correspondence: Sabrina Anne Jacob Strathclyde Institute of Pharmacy and Biomedical Sciences, University of Strathclyde, I6I Cathedral St, Glasgow, G4 ORE, UK

Tel +44 77I 8759993

Email sabrina.jacob@strath.ac.uk 
communication as there may be barriers such as surgical masks or small lips. Indeed, many participants expressed a fear of miscommunication between them and their physician, which could result in undesirable consequences such as misdiagnosis. ${ }^{7}$

Individuals who are $\mathrm{DHoH}$ generally depend on a visual language that is not available in written form. ${ }^{7,8}$ Therefore, they may not have a good grasp of written language, which makes deciphering and comprehending health information in the form of leaflets, posters, or articles, difficult. As opposed to their hearing counterparts who learn from observing and listening to conversations around them, people who are $\mathrm{DHoH}$ are deprived of incidental learning opportunities on health-related issues, having never overheard discussions of their family's medical history, or listened to radio and television commercials on health issues. Current health information provided to the general public also does not cater to patients who are $\mathrm{DHoH}$, and only a limited amount of health resources are available in sign language. ${ }^{9}$ These barriers to proper access to health care information have led to lower health literacy levels among the Deaf community, and this has translated to poorer health outcomes. ${ }^{2}$

Information and communication technology has the potential to assist marginalized social groups overcome their difficulties. ${ }^{10}$ To assist communication for individuals who are $\mathrm{DHoH}$, a number of tools have been developed to overcome language barriers. A teletypewriter allows people to type and receive messages over the phone line. However, it does not allow the sender and receiver to type at the same time, which might prove frustrating to users. ${ }^{11}$ The video relay service requires the constant presence of an interpreter, resulting in many of them experiencing high levels of stress. There have also been reports that a lag time of 60-80 milliseconds was present from the time the interpreter was translating to the time the Deaf individual perceived it. ${ }^{12}$ Further elaborations on these technologies as well as Deaf-related terms are provided in Table 1.

Mobile technologies are able to improve the health care services provided to patients and in this case bridge the gap between individuals who are $\mathrm{DHoH}$ and health care professionals. Mobile health (mHealth) is described as a tool supported by the functions of mobile devices and wireless technologies for improving an individual's overall health. ${ }^{13}$ A medication reminder app (Pill Phone) used in patients with hypertension resulted in a significant increase in adherence rates, which then translated to a significant reduction in blood pressure levels. ${ }^{14}$ Similarly the CardioManager, an app developed to help patients self-manage their cardiovascular disease, reduced the cost of management of heart failure patients by $33 \%$, and provided savings exceeding $€ 9,000$ per patient to the health care system. ${ }^{15}$ While a number of mHealth apps to assist communication between the Deaf and health care professionals have been developed in South Africa, ${ }^{16,17}$ Germany, ${ }^{18}$ and the US, ${ }^{19}$ none have been developed in Malaysia as yet.

As of 2013, there are only 30 sign language interpreters (SLI) working with the Malaysia Federation of the Deaf, which serves the 55,000 people in Malaysia who are registered as $\mathrm{DHoH}$, and have a preference for communicating in Malaysian sign language (MSL). Moreover, there are approximately 20-30 freelance interpreters across the country. ${ }^{20}$ Thus for every one interpreter, there are approximately

Table I Deaf-related terms and definitions

\begin{tabular}{|c|c|}
\hline Terms & Definitions \\
\hline Hard of Hearing $(\mathrm{HoH})$ & $\begin{array}{l}\text { Individuals with mild to moderate hearing loss who may communicate using sign language or spoken language, or } \\
\text { even both. } .^{53} \text { People with hearing loss are graded as mild, moderate, moderately severe, severe, or profound. People } \\
\text { with the latter two are referred to as Deaf, while those graded as mild or moderate are known as HoH. }{ }^{54}\end{array}$ \\
\hline Malaysian sign language (MSL) & $\begin{array}{l}\text { Primary language of the Deaf community in Malaysia. It is recognizably different from sign languages used in other } \\
\text { countries, and is heavily influenced by Malaysian culture. }{ }^{34} \text { English and Bahasa Malaysia (the national language) have a } \\
\text { different syntax from MSL. MSL sentences do not always follow subject-verb-object word order, making it difficult } \\
\text { for a Deaf person to understand written English or Bahasa Malaysia. }\end{array}$ \\
\hline Video relay services (VRS) & $\begin{array}{l}\text { Uses sign language interpreters (SLIs) to assist communication between a Deaf person who uses a 'videophone' to } \\
\text { communicate in MSL, and a hearing person who uses a standard telephone; on a computer screen through linked } \\
\text { video cameras. }\end{array}$ \\
\hline Teletypewriters (TTY) & $\begin{array}{l}\text { A device used by the Deaf to type their messages using a keyboard through telephone lines. A relay operator then } \\
\text { conveys the typed messages using either text or voice format through the telephone. }\end{array}$ \\
\hline $\begin{array}{l}\text { Video remote interpreting } \\
\text { (VRI) }\end{array}$ & $\begin{array}{l}\text { Uses video conferencing technology where SLls are remote or offsite, and communicates with the Deaf person via } \\
\text { video in MSL, but with the hearing person in audio. }\end{array}$ \\
\hline Videophone (VP) & $\begin{array}{l}\text { Deaf people who use a VRS have a phone number designated "VP". If the person they wish to call also has a VP, } \\
\text { they can call the person directly and have a video conversation in MSL. If the person they wish to call uses a voice } \\
\text { telephone, the call is routed through a VRS.7 }\end{array}$ \\
\hline
\end{tabular}

Note: This table is mirrored from a paper by Steinberg et al. ${ }^{7}$ 
1,000 Deaf individuals to attend to. Since health care professionals are not compelled to provide an SLI, patients who are DHoH often face uncertainty during their medical visits. This then highlights the need for an app that will be able to provide simple interpretations whilst reducing the need for SLIs, hence providing a far more cost-effective solution. Malaysia has satisfactory wireless broadband coverage throughout the country, although in certain rural areas this may be of poor quality. ${ }^{21}$

One of the most accessible health care professionals is a community pharmacist. Due to their close proximity and high frequency of contact with the general public, ${ }^{22}$ this places them at the point of entry to the health care system. ${ }^{23}$ Community pharmacists, therefore, can and should assume greater responsibility in the health care of patients who are DHoH. Our preliminary findings, however, indicate that the Deaf community faces communication barriers when consulting pharmacists in Malaysia. ${ }^{24}$ We have since taken the initiative to design and develop an mHealth app to facilitate communication between pharmacists and individuals who are $\mathrm{DHoH}$ and who use sign language as their primary language. To inform the design of this app, we undertook a qualitative study with the objective of obtaining the views of community pharmacists about the design and features of the app.

\section{Material and methods}

A qualitative FGD was conducted using a list of close-ended questions that was developed based on the study objectives, review of the literature, as well as information needed by Leo Burnett (Kuala Lumpur, Malaysia), the app developers (Supplementary material S1). Face and content validation was done by experts in qualitative research, and experts in app design and development. Snowball and purposive sampling was employed to obtain a target sample size of 12-15 community pharmacists, regardless of whether or not they had interacted with a patient who is $\mathrm{DHoH}$, who were able to understand and speak in English. The study was approved by the Monash University Human Research Ethics Committee (CF16/18-2016000007).

Prior to the start of the session, the explanatory statement form was given out to all participants. The objectives of the FGD were explained to the participants, and they were also notified that: the session would take 1-2 hours, their responses would be anonymized and audio-recorded, and participation in the FGD was strictly voluntary. Field notes were also taken to capture key points. Participants were then given time to ask any questions, and those who consented were then asked to sign consent forms. Participants were informed that the facilitator would pose a series of questions, and were asked to elaborate on their responses. They were also given some basic ground rules on how the FGD would proceed, namely: 1) participants were encouraged to give their opinions as freely and as honestly as they could; 2) participants were asked to allow one another a turn to talk, and 3 ) to respect the speaker by listening, not talking among themselves, and not interrupting the speaker. Participants were also requested to provide some demographic details at the end of the FGD. All participants voluntarily participated in this FGD, and were given gift vouchers amounting to USD5 to thank them for their participation.

\section{Data transcription and analysis}

Recorded interviews were transcribed verbatim, and de-identified prior to analysis. Results were then imported into NVivo 11 (QSR International, Melbourne, VIC, Australia). All audiorecordings and interviewer field notes were also imported into NVivo for comparison and analysis. Thematic analysis was performed on the transcripts by two researchers (SAJ and EC), guided by Braun and Clarke's six phase approach to coding: 1) data familiarization, 2) initial code generation, 3) theme searching, 4) reviewing of themes, 5) defining and naming themes, and 6) producing the report. ${ }^{25}$ Quotations by participants were edited on a limited basis to remove content that did not convey meaning (repeated words, stutters) and to correct for grammar. An ellipsis mark was used to note removal of such extraneous content. Square brackets were used in quotations to supply words omitted by the speaker or to replace sensitive information where names were mentioned.

\section{Results}

Twelve pharmacists consented to take part in the FGD, of which $75 \%$ were females. Saturation was achieved after the FGD, therefore no further FGDs were conducted. The majority $(83.3 \%)$ of participants had less than five years of experience as community pharmacists, while two (16.7\%) had between 10 and 14 years of experience. All participants owned a smartphone and used medical apps on their smartphones (Table 2). Thematic analysis revealed three main themes: 1) suggestions for app design and content, 2) perceived benefits of the app, and 3) potential challenges related to the app; these are summarized below and in Table 3.

\section{Theme I:Suggestions for app design and content}

\section{Information on medications and diseases}

With regard to how information about medications should be presented, most suggested having both a video in sign 
Table 2 Characteristics of participants

\begin{tabular}{|c|c|}
\hline Characteristics & $\mathbf{N}(\%)$ \\
\hline Age, years (mean $\pm S D$ ) & $32.08 \pm 5.58$ \\
\hline \multicolumn{2}{|l|}{ Role in pharmacy } \\
\hline Full-time pharmacist & $6(50.0)$ \\
\hline Locum pharmacist & $6(50.0)$ \\
\hline \multicolumn{2}{|c|}{ Number of prescriptions from Deaf or $\mathrm{HoH}$ patients } \\
\hline \multicolumn{2}{|l|}{ filled monthly } \\
\hline Never & $8(66.7)$ \\
\hline$<5$ & $4(33.3)$ \\
\hline \multicolumn{2}{|c|}{ Number of years since graduation (basic degree) } \\
\hline$<5$ & $4(33.3)$ \\
\hline $5-10$ & $5(41.7)$ \\
\hline$>10$ & $3(25.0)$ \\
\hline \multicolumn{2}{|l|}{ Type of pharmacy } \\
\hline Chain & $4(33.3)$ \\
\hline Independent & $8(66.7)$ \\
\hline \multicolumn{2}{|l|}{ Qualifications $^{\mathrm{a}}$} \\
\hline B.Pharm & II (9I.7) \\
\hline M.Pharm & $\mathrm{I}(8.3)$ \\
\hline MClinPharmacy & $2(16.7)$ \\
\hline \multicolumn{2}{|l|}{ Operating system used } \\
\hline Android & $7(58.3)$ \\
\hline iOS & $5(41.7)$ \\
\hline \multicolumn{2}{|c|}{ Frequency of using medical smartphone apps } \\
\hline $2-3$ times & $6(50.0)$ \\
\hline 4-5 times & I (8.3) \\
\hline$>5$ times & $5(4 I .7)$ \\
\hline
\end{tabular}

Note: aParticipants were allowed to select more than one response; therefore sum may be more than $100 \%$.

Abbreviation: $\mathrm{HoH}$, hard of hearing.

language with subtitles, as well as in-text descriptions, which was thought to be more comprehensive and provide better explanations. Participants also felt that the app should be interactive and dynamic, where patients have options as to what medium they would like information presented to them in ie, text format, video format, etc. Most also agreed that pictures would aid in the delivery of information to patients who are $\mathrm{DHoH}$ eg, pictures like the sun and moon to indicate morning and night. Concerns were raised, however, with regard to pictures being misinterpreted, as they were subject to interpretation by the patient.

\section{Scheduling and reminder service}

Participants suggested that patients could fill in details of their presenting complaints on the app prior to their visit to the pharmacy. Many found this useful to ease the consultation process. Some suggested including the patient's current medication information and history, such as dosage, where they are currently being treated, etc. While adding the function of a booking service to schedule/reschedule and cancel appointments was viewed favorably, many claimed that patients usually preferred to just walk in according to their own time and schedule to be monitored. Other suggestions included a pop-up to alert pharmacists when the patient requires counseling, especially for certain drugs where counseling is necessary.

\section{Notifications}

While participants preferred using in-app notifications to send messages to patients for the collection of medication, there were concerns of patients ignoring the in-app notifications, assuming it was of a trivial matter. Participants used Facebook as an example where people received a lot of irrelevant notifications all the time, resulting in most people ignoring them without even looking at them first. It was proposed instead to present the message either in picture or video form.

One participant also suggested a function that automatically sends reminders to notify patients to refill their prescriptions. This would reduce the burden on the staff of having to manually send reminders to patients all the time, which would be particularly challenging when dealing with a high volume of customers. Many believed the app should be personalized to the needs of the $\mathrm{DHoH}$, with suggestions that pop-up notifications should be in the form of sign language instead of simple texts.

\section{"Grab \& Go" service}

"Grab \& Go" is where patients purchase medications inapp - which is then prepared by the pharmacists - and collect it at a time convenient to them. Many supported the idea of a "Grab \& Go" service at community pharmacies, with one commenting it meant he would be able to spend less time talking to the patient. Participants also commented that most customers expected quick service, and as such felt that such a service would be time-saving for both pharmacists and patients. Participants suggested that patients could either scan the prescription, or take a photo of it and upload it to the app.

Participants, however, felt that the "Grab \& Go" service should be reserved for over-the-counter drugs and vitamins only and there should be a list of items that would not be permitted via the "Grab \& Go" service. Participants were also not in favor of a "delivery" option, as apart from having to incur the extra cost of postage or delivery, there were also ethical issues as it pertained to certain items. An example cited by a few participants was cough syrup, which was abused by drug addicts. As pharmacists often recognized regular drug addicts, there was the fear that drug addicts 
Table 3 Common themes identified and example responses

\begin{tabular}{|c|c|c|}
\hline Theme & Sub-theme & Examples \\
\hline \multirow[t]{4}{*}{$\begin{array}{l}\text { I. Suggestions for app } \\
\text { design }\end{array}$} & $\begin{array}{l}\text { Information on } \\
\text { medications and } \\
\text { diseases }\end{array}$ & $\begin{array}{l}\text { "The video [should come] with subtitles also. Maybe they can choose - do they want to see } \\
\text { it in video or in text because sometimes in-text [...] it is more comprehensive [...]. For those } \\
\text { who can read, for those who are literate or can read, they can really read in detail. I think it's } \\
\text { more clear rather than showing the sign language, but for those who cannot read then it's } \\
\text { better to have sign language." }\end{array}$ \\
\hline & $\begin{array}{l}\text { Scheduling and } \\
\text { reminder service }\end{array}$ & $\begin{array}{l}\text { "So actually by having the app, let's say they are able to have the app, they can just }[\ldots] \text { type } \\
\text { in what's the problem, things like that. [...] so then, if we get the information earlier, then } \\
\text { from those questions, }[\ldots] \text { when the patient is right in front of you, you can ask }[\ldots] \text { you have } \\
\text { this problem, you can actually ask more and then, you'll be ready by then [with] what kind of } \\
\text { actions you need to do or are supposed to do." }\end{array}$ \\
\hline & Notifications & $\begin{array}{l}\text { "Like something pops up into your phone }[\ldots] \text { you have to collect your medications. I think } \\
\text { that might be good. Or maybe }[\ldots] \text { it has something different from }[\text { a] message. Instead of } \\
\text { just words or what, it could come up like maybe }[a] \text { picture or maybe }[\ldots] \text { like in a video like } \\
\text { someone telling the patient }[\text { what they need to do] }[\ldots] \text { ". }\end{array}$ \\
\hline & Grab \& Go service & 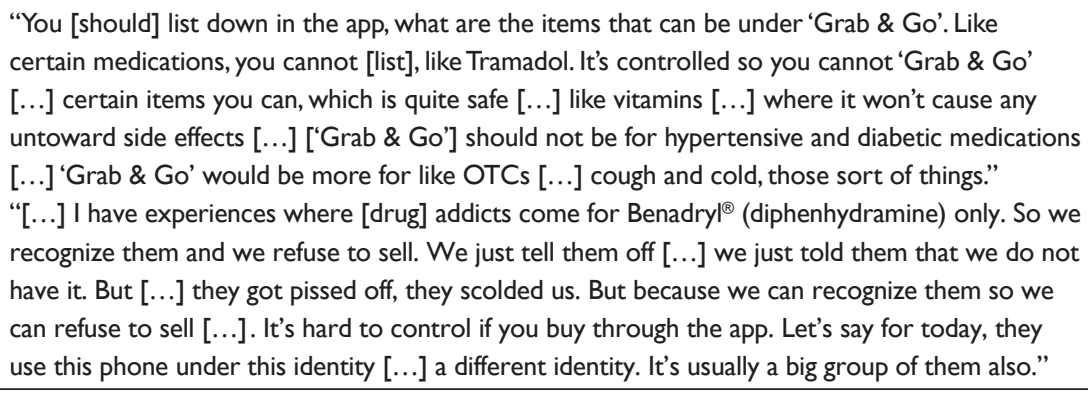 \\
\hline \multirow[t]{3}{*}{$\begin{array}{l}\text { 2. Perceived benefits of } \\
\text { the app }\end{array}$} & $\begin{array}{l}\text { Facilitates } \\
\text { communication } \\
\text { with Deaf and } \mathrm{HoH} \\
\text { patients }\end{array}$ & $\begin{array}{l}\text { "We had a patient [who came] to the discharge pharmacy who was Deaf and dumb. He was } \\
\text { illiterate and so, I had a difficult time communicating with him. He got diabetic medications } \\
\text { earlier on. Instructions were written on the envelope and I think the dispenser who dispensed } \\
\text { to him told him like one tablet once a day you know, but I think [the dispenser] couldn't } \\
\text { tell him before or after meals. So he came back again and we didn't know what he wanted } \\
\text { [...] and he got so frustrated, he showed us a picture of a policeman like saying 'l'm going to } \\
\text { complain'. So, he just couldn't [communicate] - and he got so frustrated, he walked a few } \\
\text { yards from the discharge pharmacy and yelled you know, because he was so frustrated. He just } \\
\text { yelled and yelled and yelled. You know, like crying out. He was so so frustrated." }\end{array}$ \\
\hline & Time-saving & $\begin{array}{l}\text { "[...] you can find that they want }[\ldots] \text { you to keep confirming the dose. You know like to take } \\
\text { one tablet three times a day. Yeah, I had to like repeat it to them a few times and they in turn } \\
\text { showed me like ' } I \text { ', ' } 3 \text { ' times a day (showing hand gestures). You know, like that sort of thing. } \\
\text { So it's not so [...] smooth sailing as if you had been dealing with an able-bodied person." }\end{array}$ \\
\hline & $\begin{array}{l}\text { Relationship-building } \\
\text { and boost for business }\end{array}$ & $\begin{array}{l}\text { "Yes, they will [appreciate it because they feel like they are being cared for]. They will } \\
\text { appreciate it because you know [...] they do not come out that much because of society and } \\
\text { all that. When they find, okay someone is showing them some sort of care, it's good I think." }\end{array}$ \\
\hline \multirow[t]{2}{*}{$\begin{array}{l}\text { 3. Potential challenges } \\
\text { related to the app }\end{array}$} & $\begin{array}{l}\text { Current health } \\
\text { practice in Malaysia }\end{array}$ & $\begin{array}{l}\text { "It all depends on the pharmacy itself. If the pharmacy or pharmacists care, they will [...] have } \\
\text { their own proper record in their own computer, [and] how they want to record it is up to } \\
\text { them or how to follow-up is up to them. It all depends on them, some of them just want to do } \\
\text { business so it's a totally different story." } \\
\text { "[...] I mean this app is ideal for those countries that have already established dispensing } \\
\text { separation like maybe, Australia or somewhere. But in Malaysia, I keep thinking of people } \\
\text { misusing and abusing this system." }\end{array}$ \\
\hline & App charges & $\begin{array}{l}\text { "I have an experience with an app [...] which I have used for my eye drops because I have to } \\
\text { use it 5-I0 times a day. So, it helps to remind me as and when I need to use the eye drops. } \\
\text { My initial experience with the app was okay until recently because it's been almost one month, } \\
\text { it says my trial version is ending. So if you are providing for those who are not so financially } \\
\text { well, I think we should give it for free. If we can provide that [it would be good] because there } \\
\text { are a lot of in-app purchases. You know, taking into consideration that they are illiterate and } \\
\text { not so well to do, if we really want to help them, perhaps we can think about this." }\end{array}$ \\
\hline
\end{tabular}

(Continued) 
Table 3 (Continued)

\begin{tabular}{|c|c|c|}
\hline Theme & Sub-theme & Examples \\
\hline & Privacy and security & $\begin{array}{l}\text { "Security issues. Let's say we upload it to the Cloud, it could be hacked. That could be hacked. } \\
\text { So if I were the customer, I may be worried if my information may be disclosed or exposed. } \\
\text { For example, if I buy Viagra }{ }^{\circledR} \text { then [...] right? Okay, let's say you're not uploading, the server } \\
\text { is not the Cloud, are we keeping it in the phone? If you keep it in the phone and anything } \\
\text { happens to the phone then [...] all information will be gone." } \\
\text { "Or maybe when patients key in their details, they can choose [that] they want to [...] } \\
\text { disclose this particular information to this particular shop. Maybe they want to link it, maybe } \\
\text { they want the same information going to this shop and that shop, and so only these two can } \\
\text { view their particulars." }\end{array}$ \\
\hline & $\begin{array}{l}\text { Manpower and } \\
\text { knowledge } \\
\text { requirement }\end{array}$ & $\begin{array}{l}\text { "It's good, just that it depends on whether the pharmacy has that ability or manpower. If you } \\
\text { put the feature there, and at the end [of the day] the pharmacy does not have the manpower } \\
\text { to provide that [service]. People will be disappointed. [They may ask], 'Why did you put that?"' }\end{array}$ \\
\hline & Attitude of patients & $\begin{array}{l}\text { "You can see that most of the customers that walk into the pharmacy, you can see they have } \\
\text { no patience }[\ldots] \text { and they cannot wait for you. They are in a hurry }[\ldots] \text { " }\end{array}$ \\
\hline & Patient perception & $\begin{array}{l}\text { "I think when you design the app, you have to think from two points. For example, I went } \\
\text { to a clinic and it was a newly opened clinic, and it was the first time I was seeing the doctor } \\
\text { and she gave me some consultation. It was just a sore throat and she told me 'Oh you do not } \\
\text { need antibiotics for now.' And so she discharged [me with] some medications. Then, a few } \\
\text { days later, her nurses actually called me to ask about my condition. So, I was very shocked } \\
\text { because there are several GP's l've visited and none of them have given me this type of } \\
\text { service. So that would prompt me to think, 'are you really that caring towards me or are you } \\
\text { doing it for the profit?' So I think when you are designing the aps, you need to think of this } \\
\text { because the pop-up reminders would make me felt like as if you [only] want business from } \\
\text { me, or it's time to refill so it's time to come to the pharmacy to get your medicine. That's } \\
\text { what I felt." }\end{array}$ \\
\hline
\end{tabular}

Abbreviations: GP, general practitioner; OTC, over the counter.

could abuse the app by using a fake or different identity to buy the cough syrup.

\section{Other features}

Other features recommended to be included in the app include a medical calculator to calculate body mass index, a scanner to scan prescriptions, reference ranges for various medical conditions such as blood glucose, cholesterol, etc, customers' purchase history, and a simple sign language tool to assist them in counseling. There was also a request for the details of the patients' medical history to be available, as knowledge of their allergies, and current medications would allow appropriate monitoring of the patient. With regard to language, respondents prefer one that is bilingual, with English being the preferred language for pharmacists, and Bahasa Malaysia (BM, the national language) for the individuals who are $\mathrm{DHoH}$. Participants also suggested that there should be two different interfaces in the app - one for the patient and the other for the pharmacist.

\section{Theme 2: Perceived benefits of the app Facilitates communication with patients who are $\mathrm{DHoH}$}

A number of participants commented on their difficulty in communicating with patients who are $\mathrm{DHoH}$. One participant recalled a previous experience where communication with a seemingly literate patient who was $\mathrm{DHoH}$ was very difficult, indicating a disparity in literacy level. Some found it a challenge to gather information necessary to effectively assess the patient's condition. Due to this, a number preferred writing as a mode of communication. However, even this was contingent on whether patients were literate. The presence of an interpreter was seen to be useful, but there were concerns as to the accuracy of translation. Thus, participants felt the app would help facilitate communication with patients, as they could use diagrams and pictures, or even sign language pop-ups to communicate.

\section{Time-saving}

Interactions with individuals who were $\mathrm{DHoH}$ required more time and effort as compared to their hearing counterparts. Participants believed that in many of their encounters with individuals who were $\mathrm{DHoH}$, it was a matter of guessing/ deducing what patients were trying to convey, and they attributed this to their lack of experience with this subset of patients. As such, if patients were able to fill in their complaints in the app prior to coming to the pharmacy, this could help minimize any errors due to misinterpretation, as well as the consultation time. 
The use of the "Grab \& Go" service as well would help minimize patients' waiting time, and this would satisfy the patients as most are in a hurry and want to get their medications fast. Indeed, respondents lamented the fact that they could potentially lose customers if they did not provide quick service, noting that customers get angry when not served first, and as a result, would not come back to the outlet.

\section{Relationship-building and boost for business}

Some participants felt that by sending in-app notifications or a personal text to the patients to remind them to collect their medications, this could help foster a positive relationship between the patients and the pharmacists, with patients taking it as a sign that the pharmacists have a personal interest in their health outcomes. Many also agreed that the app could display discounts and items on sale, which would boost their business.

\section{Theme 3: Potential challenges related to the app}

\section{Current health practice in Malaysia}

The majority of participants felt that there is no standardized system for patient monitoring currently in community pharmacies in Malaysia. Thus, there is no fixed system or time for a patient to come again, say after one month, to have a follow-up check. The lack of time was also highlighted as an obstacle to optimal delivery of pharmaceutical care, as pharmacists were forced to rush through counseling sessions due to the number of customers waiting in line. One commented that community pharmacies may not be able to cope with the responsibility of monitoring patients, as they found it a huge task to monitor all patients and schedule appointments.

Due to the more business-centric nature of community pharmacies, participants felt that continuity of patient care was dependent on the pharmacist. Many acknowledged the fact that chain pharmacies were more business-driven and paid little or no attention to providing pharmaceutical care. One respondent commented that while he was trained on how to push sales and sell medications in a set; no mention was made of providing pharmaceutical care to patients. Thus, the inclusion of such features in the app that prompts pharmacists on the need to monitor or counsel patients might be underutilized.

The absence of dispensing separation in Malaysia has led to some pharmacists selling medications without a prescription, and as such, participants were worried that certain pharmacists might misuse the app to sell medications. One participant also highlighted the issue of physicians prescribing without fulfilling the requirements of a valid prescription, with some being written on "sticky-notes" and some lacking details on patients' names or age altogether. This could then pose a barrier to using the app if patients are required to scan their prescriptions, as well as for legal purposes.

\section{App charges}

Providing the app at a cost would be a hindrance toward the willingness of consumers to purchase the app, given that the majority of patients who are $\mathrm{DHoH}$ come from lower socioeconomic backgrounds. Thus, there was unanimous agreement that the app should be free of charge. Participants, however, also said they would not buy the app and provide it for free to the customers, unless it meant more business for the pharmacy and was more of an investment. It was also suggested that to combat the issue of cost, the app could be included as one of the perks of membership with the pharmacy.

Participants voiced concerns that not all patients would be able to afford smartphones. They also felt that because of the features proposed, cheaper phones would not be able to run the interface properly. As such the design of the app should be a balance between simplicity and usability, to ensure a smooth user experience, and that it does not lag.

\section{Privacy and security}

There were concerns that patient confidentiality may be compromised, since patients' information stored in the Cloud may be vulnerable to hacking. On the other hand, with hardware storage, information could be lost if the hardware in question was lost. There were also concerns about privacy settings - if competitor pharmacies would be able to view their patient information. To combat this issue, participants suggested that patients be given the option of whether they wished to disclose certain personal information to a particular pharmacy, while keeping it hidden from another pharmacy. Another suggestion was that each pharmacy had its own ID and patients were given IDs specific to the pharmacy they frequented. This would then prevent other pharmacies from having access to information such as purchase history, from other pharmacies the patients frequented.

\section{Manpower and knowledge requirement}

A major drawback of the app is that it will require time, commitment, and manpower to manage certain features of the app such as the quick-chat tools, and support groups. As most pharmacies only had one pharmacist at a time, they would not have the time to answer questions, and as 
such there would be a need to hire dedicated personnel to respond to queries. They also felt it would be difficult trying to live up to patients' expectations to respond to their queries within a short period. Participants unanimously rejected the idea of support groups, due to the time constraints as well as the worry that they lacked the knowledge and experience to handle patients' queries, especially as it pertained to topics they were not experienced in.

\section{Attitude of patients}

With regard to including functions that alert pharmacists on when patients should be monitored, or when counseling should be provided, many participants felt the patient's attitude would be the biggest barrier to the use of this function as patients were often in a hurry to get their medications. Furthermore, respondents were concerned that patients who do not like going to the hospital might not feel the need to continue their follow-ups at hospitals if the pharmacists provided frequent monitoring.

\section{Patient perception}

Some participants highlighted the fact that the app, particularly the "Grab \& Go" feature, might not be well received by all patients, with some perceiving it as being impersonal. This they felt depended on location as well as the education level of the patients. Participants commented that customers from urban areas and with a higher level of education would appreciate the app more, compared to customers from rural areas who generally prefer to come in to the pharmacy to sit and talk. There were also concerns that patients might perceive the app and notifications not as tools to help them, but rather a way for the pharmacists to increase their revenue. This would then turn them off from visiting the pharmacy again.

\section{Discussion}

This study is the first of its kind to be conducted in Malaysia. Overall, our participants positively perceived the potential app, and the opportunity for the app to enhance communication between pharmacists and patients who are $\mathrm{DHoH}$ was acknowledged. Many emphasized the importance of personalizing the app to the needs of the Deaf. Based on the findings of the FGD, three main themes were identified and are discussed below.

\section{Suggestions for app design}

Participants agreed that providing information in video form with texts would be helpful for Deaf patients. Chininthorn et al noted that an effective technique of delivering information to people who are $\mathrm{DHoH}$ is through pictures or videos with simple text descriptions, as it strengthens their understanding of diseases and medications. ${ }^{16}$ As individuals who are $\mathrm{DHoH}$ are visual learners and thinkers, illustrations and animations would help improve their retention of information, and pique their interest. ${ }^{26}$ Similar to our participants, Deaf health care professionals, and policymakers in South Africa agreed that providing health information in their local sign language was essential for the Deaf community. ${ }^{16}$ However, visual perception varies from person to person, ${ }^{27}$ therefore, misinterpretations and ambiguity over pictures may occur. It was suggested that this can be overcome by including side-by-side captions to provide more context to the pictures. ${ }^{16}$

In South Africa, an app called SignSupport was developed to facilitate communication between individuals who are $\mathrm{DHoH}$ and health care professionals. The app had the capability of prompting the pharmacist to provide instructions on medications by selecting the provided options. These options included pre-set South African sign language (SASL) recordings that can be constructed into a video to counsel the patients based on their medications, ${ }^{16}$ text to image/video clips, and translations from English text to SASL. ${ }^{28}$ Deaf patients reported that SignSupport was accessible, affordable, and comprehensible during user trials, while pharmacists felt the app helped them fulfill their professional obligations. ${ }^{28}$ This can be adapted to the proposed app to facilitate patient counseling.

The scheduling and reminder feature was well-accepted by participants. The rate of deafness increases with age, starting from $2 \%$ in adults aged $45-54$, to $50 \%$ by the time they are above 70 years old. ${ }^{29}$ Indeed, it is estimated that one third of the world's population over the age of 65 experience disabling hearing loss. ${ }^{1}$ Malaysia has 2.8 million senior citizens aged 60 and above, and is anticipated to reach aging population status by $2035 .^{30}$ Aging also comes with memory decline, and this is a major barrier to medication adherence. ${ }^{31}$ As such, having the schedule and medication reminder features in the app would be useful when nonadherence is unintentional. Since older people have complex treatment regimens and experience forgetfulness, the usage of the app together with their pillbox devices would provide additional benefit.

Studies, however, have shown that achieving better longterm adherence and clinical outcomes are most effective through intricate combinations and multiple strategies like identifying the reasons behind nonadherence, and including 
behavioral models such as motivational interviewing. ${ }^{32,33}$ Thus, pharmacists need to be cognizant of the fact that the app is not a substitute for proper counseling sessions to address intentional adherence. Many elderly patients are also not technologically-savvy, thus the impact of usability in the elderly should be taken into consideration. As such, app creators need to include large fonts, clear buttons, contrasting text, and clear instructions to accommodate this age group. ${ }^{33}$

With regard to language use in the app, participants suggested that $\mathrm{BM}$ be used for the $\mathrm{DHoH}$. This highlights a common misconception that sign language is a direct translation from the spoken language. The vocabulary and syntax in sign language is in fact distinct from the spoken language, and the linguistic aspects of visual sign language greatly differ from spoken language. ${ }^{9}$ Sign language also differs from country to country and cannot be generalized as a universal language, ${ }^{34}$ thus the Deaf in Malaysia use MSL, which is different from spoken BM. It is, therefore, important to educate pharmacists on the differences between MSL and $\mathrm{BM}$ to prevent miscommunication from occurring. As many studies have emphasized the importance of embracing Deaf culture, ${ }^{2-4}$ incorporating both MSL and BM in the app would be vital to ensure acceptance by the $\mathrm{DHoH}$ community.

\section{Perceived benefits of the app}

Participants felt the app could potentially save time, and allow for a more efficient dispensing process. In trial-runs using SignSupport, the average dispensing time was 4:23 minutes per patient vs 19:55 minutes per patient without the app. ${ }^{35}$ This is probably due to the lower literacy levels among the Deaf, where it has been noted that the average reading level of English among Deaf individuals using ASL is usually at sixth grade or below, which makes the use of written communication difficult. ${ }^{9}$ Since patients will be able to detail their medical complaints via the app prior to their visit to the pharmacy, less time would be needed to gather information and dispense the medication.

Physicians have reported that Deaf individuals had great difficulty in understanding them, and maintaining a conversation. They also reported uneasiness when treating Deaf patients, which may have been due to the lack of understanding of Deaf culture ${ }^{36}$ and the limited knowledge on issues related to the Deaf. ${ }^{37}$ With the app, health care professionals will be better able to deliver their counseling or dispensing with the aid of pictures. The app would also be able to indirectly educate health care professionals on Deaf culture, and improve their attitude toward the Deaf.
Participants also felt the app could be beneficial from a business standpoint. In 2015, it was reported that the time spent on mobile apps had exceeded the time people spent watching television in the USA. ${ }^{38}$ With the increased usage of apps worldwide, displaying advertisements or deals-ofthe-week on the app may increase business for the pharmacy. Push notifications can alert customers regarding items on sale, and the pharmacy's upcoming events anywhere and at any time. This would increase customer engagement, and provide a more efficient and cheaper method of marketing compared to handing out flyers or sending emails.

\section{Potential challenges related to the app}

It is not uncommon to see only one pharmacist present at a community pharmacy at any given time, which explains why a number of studies have highlighted the same barriers to providing pharmaceutical care as our participants, such as limited time, the lack of trained personnel, and the lack of motivation. ${ }^{39-41}$ Previously, provisionally registered pharmacists (PRP) in Malaysia were only allowed to do their one-year training in the government setting. In 2012, the Malaysian government allowed PRP training to be carried out at community pharmacies as well, ${ }^{42}$ thus this would overcome the issue of limited manpower.

From our findings, participants admitted that chain pharmacies usually overlooked pharmaceutical care, and were more concerned with profit. However, customer satisfaction has been shown to increase with pharmaceutical care playing a role in improving the quality of health care, and in turn, boosting the financial returns of the business through a perpetual group of loyal customers. ${ }^{43}$ Thus, pharmacists can leverage the app to provide pharmaceutical care services to patients, which would increase adherence rates, and subsequently increase the purchase of drugs.

The majority of our participants requested for the app to be free of charge, noting that Deaf adults are often unemployed and for those who are, they are in the lower ranks of employment compared to the general public. ${ }^{44}$ Thus, providing the app at a cost may deter them from using it. Another alternative would be to provide the app for free but derive revenue from advertisements. ${ }^{45}$ It was found that about $89 \%$ of global app downloads in 2012 were free, ${ }^{46}$ and perceived value, app-ratings, free options of paid apps, and patient satisfaction displayed by potential and actual users, had a large influence on the intention to pay for an app. ${ }^{45}$ Participants also noted that the app must have the ability to function on a spectrum of smartphone models from low-cost to high-end ones. Although smartphones are currently not 
very cheap, manufacturers are moving toward producing more affordable options. ${ }^{47}$

Privacy was a major concern for the participants, as mHealth apps store not just personal data, but also information on the user's health such as allergies, past medication history, and past medical history. Data inserted into the app is often valuable, as well as vulnerable to hackers. The privacy and security of apps available in Canada were explored, and it was discovered that out of 184 free mHealth apps evaluated, only $34.2 \%$ provided a privacy policy, $70.1 \%$ did not have password protection, and only 20 out of 184 apps provided private alerts. The authors suggested that the possibility of security and privacy breach could be avoided by ensuring there is a password or a log-in system through an email and password. Information stored should be encrypted, and there should also be an option to have private notifications which do not display the medication name. A remote wipe and a timeout after a few unsuccessful attempts of the password could also be included in the app. ${ }^{48}$ Customization of privacy settings would also be helpful as participants voiced that patients who were Deaf should have the ability to control what information should be disclosed and to whom. ${ }^{49}$

The negative attitude of patients was another barrier identified, where patients want their medications quickly and are not interested in pharmaceutical care. Patients, especially the Deaf, may be unfamiliar with the new concept of pharmaceutical care, which encompasses more than just the traditional roles of dispensing and compounding medications. A study involving 103 participants living in rural areas in the UK indicated that many still lacked the awareness on the services that a community pharmacist could provide, with $86.4 \%$ stating that the primary role of a pharmacist was to dispense medication. None of the participants knew that other programs like the diabetes health check program, were available in pharmacies. ${ }^{50}$

Similarly, the public's attitudes toward community pharmacies in Qatar was evaluated, where $70 \%$ of the participants $(\mathrm{N}=58)$ disagreed that pharmacists were expected to conduct patient monitoring to ensure the safety of medication use, and that pharmacists had a role in performing screening and monitoring on diseases. ${ }^{51}$ Therefore, conveying this information on pharmaceutical services is vital if the potential benefits to the Deaf community are to be realized. Just as important, the public should be made aware of the concept of pharmaceutical care, in order to get the full benefit of the app. Although the app may help to increase the awareness of the services through the notifications, Wood et al noted that community pharmacists still need to take on the active role of promoting these services to their clientele. ${ }^{52}$

\section{Strengths and limitations}

This is the first study of its kind where the views of pharmacists, who would eventually be one of the end users, were sought to inform the design of an app intended to improve their communication with patients who are $\mathrm{DHoH}$. However, all participants were pharmacists practicing in urban areas, thus this might not provide a true depiction of the potential issues related to the app that might be faced by those practicing in rural areas. In addition, a large number of participants had less than five years of experience as community pharmacists, and they might not have had enough experience dealing with patients who are $\mathrm{DHoH}$ or with communication disabilities. Data obtained here will be combined with an FGD involving the Deaf community, to inform the design of the mHealth app.

\section{Conclusion}

Through the development of this app, it is anticipated that individuals who are $\mathrm{DHoH}$ would be better served with improved communication, which will hopefully translate to better health outcomes and improved patient satisfaction. From the pharmacists' perspective, the app will facilitate a better understanding of Deaf culture and assist in providing pharmaceutical care to these patients.

\section{Acknowledgments}

The authors would to thank all of the retail pharmacists who gave their time to take part in this study. This study was funded by the Health and Wellbeing Cluster, Global Asia in the 21st Century (GA21) Platform at Monash University Malaysia. Partial publication funding was received from Leo Burnett Malaysia, HumanKind experience research. The funders had no role in study design, data collection and analysis, decision to publish, or preparation of the manuscript.

\section{Disclosure}

The authors report no conflicts of interest in this work.

\section{References}

1. World Health Organization. WHO global estimates on prevalence of hearing loss. Mortality and burden of diseases and prevention of blindness and deafness 2012. Available from: http://www.who. int/pbd/deafness/WHO_GE_HL.pdf?ua=1. Accessed December 4, 2016.

2. Hoang L, Lahousse SF, Nakaji MC, Sadler GR. Assessing deaf cultural competency of physicians and medical students. J Cancer Educ. 2011;26(1):175-182. 
3. Kuenburg A, Fellinger P, Fellinger J. Health care access among Deaf people. J Deaf Stud Deaf Educ. 2016;21(1):1-10.

4. Ferguson M, Liu M. Communication needs of patients with altered hearing ability: Informing pharmacists' patient care services through focus groups. J Am Pharm Assoc. 2015;55(2):153-160.

5. Hyoguchi N, Kobayashi D, Kubota T, Shimazoe T. Effects on Deaf patients of medication education by pharmacists. J Deaf Stud Deaf Educ. 2016;21(4):416-421.

6. Smith SR, Samar VJ. Dimensions of Deaf/hard-of-hearing and hearing adolescents' health literacy and health knowledge. J Health Commun. 2016;21(Supp2):141-154.

7. Steinberg AG, Barnett S, Meador HE, Wiggins EA, Zazove P. Health care system accessibility. Experiences and perceptions of deaf people. J Gen Intern Med. 2006;21(3):260-266.

8. Australia D.C. Information sheets - what is Auslan? 2018. Available from: https://deafchildrenaustralia.org.au/information-sheets-what-isauslan/. Accessed September 25, 2018.

9. McKee MM, Paasche-Orlow MK, Winters PC, et al. Assessing health literacy in Deaf American sign language users. J Health Commun. 2015;20(Suppl 2):92-100.

10. Valentine G, Skelton T. 'An umbilical cord to the world'. Info Commun Soc. 2009;12(1):44-65.

11. Saladin SP, Hansmann SE. Psychosocial variables related to the adoption of video relay services among deaf or hard-of-hearing employees at the Texas School for the Deaf. Assist Technol. 2008;20(1):36-47.

12. Bower K. Stress and burnout in video relay services (VRS) interpreting. J Interpretation. 2015;24(1):1-16.

13. Agarwal S, Lefevre AE, Lee J, et al. Guidelines for reporting of health interventions using mobile phones: mobile health (mHealth) evidence reporting and assessment (mERA) checklist. BMJ. 2016;352:11174.

14. Patel S, Jacobus-Kantor L, Marshall L, et al. Mobilizing your medications: an automated medication reminder application for mobile phones and hypertension medication adherence in a high-risk urban population J Diabetes Sci Technol. 2013;7(3):630-639.

15. Cano Martín JA, Martínez-Pérez B, de La Torre-Díez I, LópezCoronado M. Economic impact assessment from the use of a mobile app for the self-management of heart diseases by patients with heart failure in a Spanish region. J Med Syst. 2014;38(9):96.

16. Chininthorn P, Glaser M, Tucker WD, Diehl JC. Exploration of Deaf people's health information sources and techniques for information delivery in Cape Town: a qualitative study for the design and development of a mobile health app. JMIR Hum Factors. 2016;3(2):e28.

17. Chininthorn P. SignSupport [homepage]; 2016. Available from: https:// www.signsupport.org/. Accessed June 30, 2017.

18. iSignIT. App synopsis; 2013. Available from: http://isignit.weebly. com/app-synopsis.html. Accessed June 30, 2017.

19. Ava. Ava For Professionals; 2016. Available from: https://www.ava. me/about/. Accessed June 30, 2017.

20. Murad D. MFD: Massive shortage of sign language interpreters. The Star Online. 2013 Sep 20; Available from: https://www.thestar.com.my/ news/nation/2013/09/20/more-interpreters-needed/\#6893GkV3L 39suEc4.99. Accessed June 30, 2017.

21. Malaysian Communications and Multimedia Commission. Network performance report 2017: Measuring Malaysia broadband and voice performance. 2018. Available from: https://www.skmm.gov.my/ skmmgovmy/media/General/pdf/MCMC-Network-Performance-QoSReport-2017-final-1Feb2018.pdf. Accessed June 30, 2017.

22. Shafie AA, Hassali MA. Community pharmacy dispensing costs in the state of Penang, Malaysia. J Appl Pharm Sci. 2011;1(6):143.

23. World Health Organization. The role of pharmacists in the healthcare system. 1997:51. Available from: http://apps.who.int/medicinedocs/ pdf/h2995e/h2995e.pdf. Accessed August 7, 2017.

24. Jacob SA, Chin JR, Ying Qi T, Palanisamy UD. The needs of the deaf and hard of hearing when seeking pharmaceutical care. Res Social Adm Pharm. 2016;12(4):664-665.

25. Braun V, Clarke V. Using thematic analysis in psychology. Qual Res Psychol. 2006;3(2):77-101.
26. Obosu GK, Adu-Agyem J, Opoku-Asare NA. The use of visual art forms in teaching and learning in schools for the deaf in Ghana: investigating the practice. Int J Study Devel. 2013;2(5):408-422.

27. Carbon CC. Understanding human perception by human-made illusions. Front Hum Neurosci. 2014;8:566.

28. Blake E, Tucker W, Glaser M. Towards communication and information access for Deaf people. South Afr Comput J. 2014;54(2): $10-19$.

29. National Institute on Deafness and Other Communication Diseases Disorders (NIDCD). Quick statistics about hearing. Statistics and Epidemiology; 2016. Available from: https://www.nidcd.nih.gov/health/ statistics/quick-statistics-hearing. Accessed June 20, 2017.

30. The Sun Daily. Senior citizens make up $2.8 \mathrm{~m}$ of Malaysia's population. The Sun Daily. 2016;2016. Available from: https://www.thesundaily. my/archive/1737046-BSARCH356550. Accessed June 2, 2018.

31. Yap AF, Thirumoorthy T, Kwan YH. Medication adherence in the elderly. J Clin Gerontol Geriatr. 2016;7(2):64-67.

32. Dayer L, Heldenbrand S, Anderson P, Gubbins PO, Martin BC. Smartphone medication adherence apps: potential benefits to patients and providers. J Am Pharm Assoc. 2013;53(2):172-181.

33. Grindrod KA, Li M, Gates A. Evaluating user perceptions of mobile medication management applications with older adults: a usability study. JMIR Mhealth Uhealth. 2014;2(1):e11.

34. World Federation of the Deaf. WFD Statement on Standardized Sign Language. Available from: http://wfdeaf.org/news/wfd-statement-onstandardized-sign-language/. Accessed September 13, 2018.

35. Motlhabi MB, Glaser M, Tucker WM. SignSupport: a limited communication domain mobile aid for a Deaf patient at the pharmacy. Southern African Telecommunication Networks and Applications Conference; 2013; Stellenbosch, South Africa.

36. Ralston E, Zazove P, Gorenflo DW. Physicians' attitudes and beliefs about deaf patients. J Am Board Fam Pract. 1996;9(3):167-173.

37. Velonaki VS, Kampouroglou G, Velonaki M, Dimakopoulou K, Sourtzi P, Kalokerinou A. Nurses' knowledge, attitudes and behavior toward Deaf patients. Disabil Health J. 2015;8(1):109-117.

38. Beaver L. US consumers are now spending more time on apps than watching TV; 2015. Available from: http://www.businessinsider.com/ us-is-spending-more-time-on-apps-than-tv-2015-9/?r=AU\&IR=T Accessed June 26, 2017

39. Uema SA, Vega EM, Armando PD, Fontana D. Barriers to pharmaceutical care in Argentina. Pharm World Sci. 2008;30(3):211-215.

40. Okonta JM, Okonta EO, Ofoegbu TC, Mathew J, Oboma E, Chimuaya T. Barriers to implementation of pharmaceutical care by pharmacists in Nsukka and Enugu metropolis of Enugu state. J Basic Clin Pharm. 2012;3(2):295-298.

41. Rana Mohammed Ghazal RMG, Ghazal RM, Hassan NAG, Ghaleb O, Ahdab A, Saliem II. Barriers to the implementation of pharmaceutical care into the UAE community pharmacies. IOSR J Pharm. 2014;04(05): 68-74.

42. Malaysian Pharmaceutical Society. Liberalisation of PRP Training. 2012; 30 March 2012. Available from: http://www.mps.org.my/newsmaster.cfm? \&menuid=37\&action=view\&retrieveid=3599. Accessed May 10, 2017

43. Minarikova D, Malovecká I, Foltán V. Patient/customer satisfaction with pharmaceutical care in community pharmacies. Social pharmacy: state, problems and perspective 2014; National University of Pharmacy, Kharkiv, Ukraine.

44. World Health Organization. Deafness and hearing loss; 2017. Available from: http://www.who.int/mediacentre/factsheets/fs300/en/. Accessed July 9,2017

45. Hsu CL, Lin JCC. What drives purchase intention for paid mobile apps? - An expectation confirmation model with perceived value. Electron Commer Res Appl. 2015;14(1):46-57.

46. Gartner Inc. Gartner says free apps will account for nearly 90 percent of total mobile app store downloads in 2012; 2012. Available from: http://www.gartner.com/newsroom/id/2153215. Accessed June 30, 2017. 
47. Williamson SS, Gorman PN, Jimison HB. A mobile/web app for long distance caregivers of older adults: functional requirements and design implications from a user centered design process. AMIA Annu Symp Proc. 2014;2014:1960-1969.

48. Grindrod K, Boersema J, Waked K, Smith V, Yang J, Gebotys C. Locking it down: The privacy and security of mobile medication apps. Can Pharm J. 2017;150(1):60-66.

49. Ramanathan N, Swendeman D, Comulada WS, Estrin D, RotheramBorus MJ. Identifying preferences for mobile health applications for self-monitoring and self-management: focus group findings from HIVpositive persons and young mothers. Int J Med Inform. 2013;82(4): e38-e46.

50. Merks P, Świeczkowski D, Jaguszewski MJ. Patients' perception of pharmaceutical services available in a community pharmacy among patients living in a rural area of the United Kingdom. Pharm Pract. 2016; 14(3):774.
51. El Hajj MS, Salem S, Mansoor H. Public's attitudes towards community pharmacy in Qatar: a pilot study. Patient Prefer Adherence. 2011; 5:405-422.

52. Wood K, Gibson F, Radley A, Williams B. Pharmaceutical care of older people: what do older people want from community pharmacy? Int J Pharm Pract. 2015;23(2):121-130.

53. Padden C, Humphries T. Deaf In America: Voices from a Culture. United States of America: Harvard University Press; 1988.

54. Smith RJH, Bale JF, White KR. Sensorineural hearing loss in children. Lancet. 2005;365(9462):879-890. 


\section{Supplementary material \\ Supplementary material SI}

Interview questions

1. Would you be open to providing counseling or giving advice on medication through a video call?

2. Do you think it will ease the consultation/prescription process if patients fill forms prior to their visit?

3. Would you prefer to send an in-app notification or a personal text to the patients for collection of their medication?

4. If patients are uncertain about anything related to their medication eg, what to do in case of missed doses, contraindications, dosage etc, would you prefer providing a text description or image/video directions?

5. Would you utilize/recommend a booking service to schedule, reschedule and cancel appointments?

6. Would you offer a grab and go service at your pharmacy? If you don't own the pharmacy, would you support/ recommend offering such a service? Note: Where patients can purchase medications in-app and collect from the directed pharmacy.

7. If patients are uncertain about anything related to their medication eg, what to do in case of missed doses, contraindications, dosage etc, would you utilize a quick chat tool on an app?
8. Have you used in-app services before?

- What in your opinion are the advantages and disadvantages of in-app services?

9. Are visuals a key factor even when the content is the same?

10. Would you participate in community-based interaction? For example, support groups, etc.

11. Would you offer delivery services? (One time/weekly/ monthly plan.) If you don't own the pharmacy, would you support/recommend offering such a service?

12. What kind of features would you like to see/include in a proposed app? (This can include information on medication eg, side effects, drug interactions, indications etc; monitoring required; apt dates; refill reminders.)

13. Any specific interfaces/design that you would like to see/use in the app?

- Any ones or examples that you are currently using that you find interesting that we can adapt?

14. Will the cost be a factor when purchasing apps?

- How much are you willing to pay for such an app?

- Would your pharmacy provide such a service for free?

- Should your pharmacy provide such a service for free?

\section{Publish your work in this journal}

Patient Preference and Adherence is an international, peer-reviewed, open access journal that focuses on the growing importance of patient preference and adherence throughout the therapeutic continuum. Patient satisfaction, acceptability, quality of life, compliance, persistence and their role in developing new therapeutic modalities and compounds to optimize clinical outcomes for existing disease states are major areas of interest for the journal. This journal has been accepted for indexing on PubMed Central. The manuscript management system is completely online and includes a very quick and fair peer-review system, which is all easy to use. Visit http://www. dovepress.com/testimonials.php to read real quotes from published authors. 